East African Medical Journal Vol. 78 No. 9 September 2001

INCIDENCE AND CHARACTERISTICS OF MYASTHENIA GRAVIS IN DAR ES SALAAM, TANZANIA

W.B.P. Matuja, MBChB, MRPI, E.A. Aris, MD. MMed, Neurology, Department of Internal Medicine, J. Gabone, MD, MMed, Department of Diagnostic

Radiology and E.M. Mgaya, MD, MMed, Department of Histopathology, Muhimbili University College of Health Sciences, University of Dar es Salatan

Request for reprints to: Professsor W.B.P. Matuja. Neurology Unit, Department of Internal Medicine, P.O. Box 6500I, Dar es Salaam

\title{
INCIDENCE AND CHARACTERISTICS OF MYASTHENIA GRAVIS IN DAR ES SALAAM, TANZANIA
}

\author{
W.B.P. MATUJA, E.A. ARIS, J. GABONE and E.M. MGAYA
}

\begin{abstract}
Objective: To ascertain the annual incidence and characterise the clinical features of Myasthenia gravis in Dar es Salaam, Tanzania during a ten-year period from 1st January 1988 to 31 December 1998.

Design: Prospective cumulative registration at a major urban hospital of all patients with newly diagnosed Myasthenia gravis who were resident in Dar es salaam.

Setting: Muhimbili Medical Centre Teaching Hospital, Dar es Salaam, Tanzania.

Subjects: Forty seven patients, twenty males, twenty seven females satisfied the criteria for the diagnosis of Myasthenia gravis (MG).

Results: The annual incidence of MG of both sexes was 3 per 1,000,000 population of all age groups $(95 \% \mathrm{CI} 2.0$ to 3.6). The incidence per $1,000,000$ population was lowest in those aged below ten years 2.2 (95\% CI 1.4 to 3.4$)$ which was statistically significant. The incidence per $1,000,000$ per year was higher in females but this was not statistically significant. The clinical presentation of MG in Tanzania was localised disease accounting for $47 \%$ ocular type and $53 \%$ was mild to moderately severe generalised type MG. Twenty per cent of patients with generalised MG presented with bulbar features. Single fibre electromyography was the most sensitive test.

Conclusion: Myasthenia gravis is fairly rare in Tanzania as is in other sub-Saharan countries and presents in similar way to European in terms of age, sex, and clinical features. Ocular Myasthenia gravis was more prevalent in Tanzania than in Europe.
\end{abstract}

\section{INTRODUCTION}

Myasthenia gravis (MG) is a rare disease of the neuromuscular junction, which afflicts all races. It is characterised by weakness of skeletal muscles, which increases with muscle activity. The prevalence in developed countries range from one in 50,000 to one in 10,000 of population(1). The disease affects young adults with preponderance of females than males(1).

Myasthenia gravis seems to be extremely rare in Africans based on the scant bibliography published(2-5). In a hospital-based study, the incidence of MG in Libya adjusted for average was 4.4 persons per million per year, which was lower than those in developed countries(4). Nevertheless, the sex and age incidence of MG in Africans was similar to those in developed countries $(4,5)$. There is considerable delay from the onset of symptoms to the time of diagnosis in African patients with MG(5).

To the best of our knowledge, we are not aware of descriptive case studies in MG in East Africa. The purpose of this study was to describe the clinical characteristics and incidence of MG in Africans as seen in Dar es Salaam city.

\section{MATERIALS AND METHODS}

This study was conducted at Muhimbili Medical Centre (MMC), the only referral hospital in Dar es Salaam, which has an estimated population of 2.3 million inhabitants(6).

The study included patients referred to the neurology unit between 1st January 1988 and 31st December 1998 with provisional diagnosis of Myasthenia gravis or myopathy. The study was designed to identify and characterise the clinical presentation of Myasthenia gravis in an African population.

To ensure completeness of ascertainment, all three governmental district and three major non-governmental hospitals were visited each year by WBPM, one of the authors, to scrutinise records of patients and who later saw those patients with possible diagnosis of MG. During such visits, doctors in these hospitals were reminded to refer all patients who presented with muscle weakness due to suspected muscle diseases.

The diagnostic work up included a detailed medical history using a semi structured and standardised questionnaire with emphasis on variation of muscle weakness or fatigue of muscles. A detailed general, systems and neurological assessment were performed on each patient. Muscle fatigue was tested by sustained activity in the affected individual muscles. Clinical stage of the disease was based on Osserman's classification(7). 
A formal written consent was obtained on each patient for an edrophonium hydrochloride test. After focussing on one or more unequivocally weak muscles groups, an initial dose of $2 \mathrm{mg}$ edrophonium was given intravenously. If definite improvement occurred, the test was considered positive and terminated. If there was no response, the patient was given an additional $8 \mathrm{mg}$ and evaluated. The inclusion criteria were: (i) resident of Dar es Salaan; (ii) positive fatigue test and; (iii) objective positive response to edrophonium hydrochloride, while the exclusion criteria were: (i) non-resident of Dar es Salaam; (ii) equivocal or negative test to edrophonium hydrochloride and; (iii) muscle wasting.

All patients who fulfilled the inclusion criteria were invited for further investigations and gave written consent to the following: (i) nerve conduction by recording compound action motor potential of the ulnar nerve by supramaximal intensity repetitive stimuli at the elbow, at a rate of two to three per second of two muscles supplied by this nerve using Belly tendon recording technique or facial nerve with at least two muscles innervated by this nerve. A decrement response of more than $10 \%$ between first and the smallest of first five stimuli was recorded as positive; (ii) electromyography of selected muscle using bipolar concentric needle to examine for insertional activity, spontaneous activity, motor unit potentials and recruitment and interference pattern. Todetermine Single Fibre Electromyography (SFEM), a single fibre needle was inserted into Brachioradialis muscle to determine electromyography jitter by axonal stimulation. Values of mean consecutive differences were obtained; (iii) muscle biopsy of either the biceps or quadriceps muscle was performed on patients with generalised muscle involvement. A simple routine staining with haematoxylin and eosin for histological analysis of muscle size, cellular infiltration was carried out; (iv) chest-x-ray including view of the mediasternum and; (v) computer tomography of the chest for patients who were recruited in the study from 1996 when the CT scan facility became available.

Statistical analysis: Data were entered in computer data sheet SPSS. Chi-square tests and Student's t-test was used to compare baseline characteristics. In calculating the incidence data the 1988 national population census were used for projection of populations for each year up to 1998 using a geometric growth model. The number of population in age groups $0-19,20-39,40$ -
59 and 60 years and above were noted and the difference in population numbers in each group between 1988 and 1998 was calculated. The estimated mean numbers of subjects in the age groups $0-19,20-39,40-59,60$ and above, during the years of the study were $863000,617000,180000$ and 58400 , respectively. All $95 \%$ confidence intervals for incidence are based on the Poisson distribution.

\section{RESULTS}

During the ten years, 1988 and 1998 inclusive, 59 cases were referred to the neurology clinic. Forty seven cases satisfied the criteria for clinical diagnosis of MG and were subjected to analysis. Table 1 shows the number of patients seen in each age group each year. Of the 47 patients, 23 were seen in the first five years and 24 in the second five years. Only two patients were children below the age of ten and only two patients were 60 years and above.

The average crude annual incidence of diagnosed Myasthenia gravis over the ten-year period for both sexes was three per $1,000,000$ population per year $(95 \%$ confidence interval 2.0 to 3.6). The incidence per $1,000,000$ per year in different age groups was 2.2 (1.4 to 3.4$)$ in the 0-19 age group, $3.2(2.0$ to 4.9$)$ at $20-39.3 .3(1.4$ to 6.8$)$ at 40-59 and $3.4(0.6$ to 11.3) at 60 years and above.

The peak sex incidence for females was in the second decade and there was no patient aged above 39 years. The peak incidence for males was in the third decade. The female/male sex incidence ratio was 1.35:1.

The characteristics of the patients are shown in Table 2. Twenty seven $(57.5 \%)$ of the patients were females and $20(42.5 \%)$ were males giving a female to male ratio of 1.35:1. The mean age for all patients was 26.4 years with a range of 4 to 62 years. The mean age for females was 21 years with a range of 4 to 39 years and the mean age for males was 32.2 years, with a range of 13 to 62 years.

Table 1

Number of patients with newly diagnosed MG between 1st January 1988 to 31 st December 1998

\begin{tabular}{|c|c|c|c|c|c|c|c|c|}
\hline \multirow[b]{2}{*}{ Year } & \multicolumn{6}{|c|}{ Age (years) } & \multirow[b]{2}{*}{60 and $>$} & \multirow[b]{2}{*}{ Total } \\
\hline & $0-9$ & $10-19$ & $20-29$ & $30-39$ & $40-49$ & $50-59$ & & \\
\hline 1988 & 2 & l & - & 1 & - & - & - & 4 \\
\hline 1989 & - & - & - & - & - & - & - & - \\
\hline 1990 & - & - & 2 & 1 & - & - & - & 3 \\
\hline 1991 & - & 1 & - & - & - & - & - & 1 \\
\hline 1992 & - & - & 1 & 2 & 1 & 1 & 1 & 6 \\
\hline 1993 & - & 4 & 2 & 2 & - & 1 & - & 9 \\
\hline 1994 & - & 5 & 3 & - & - & 1 & - & 9 \\
\hline 1995 & - & - & - & - & 1 & - & - & 1 \\
\hline 1996 & - & - & - & - & - & - & 1 & 1 \\
\hline 1997 & - & 5 & 1 & 2 & 1 & - & - & 9 \\
\hline 1998 & - & 1 & 2 & 1 & - & - & - & 4 \\
\hline Total & 2 & 17 & 11 & 9 & 3 & 3 & 2 & 47 \\
\hline
\end{tabular}


Table 2

Characteristics of patient wit $M G$

\begin{tabular}{|c|c|c|}
\hline . & $\mathrm{N}=47(\%)$ & $P$ value \\
\hline Sex: Female & $27(57.5)$ & \\
\hline Male & $20(42.5)$ & \\
\hline Age: Total range & $4-62$ & \\
\hline Mean & 26.4 & \\
\hline \multicolumn{3}{|l|}{ Female: } \\
\hline Range & $4-39$ & 0.000 \\
\hline Mean & $2 !$ & \\
\hline \multicolumn{3}{|l|}{ Males: } \\
\hline Range & $1.3-62$ & \\
\hline Mean & 34.2 & 0.000 \\
\hline \multicolumn{3}{|c|}{ Duration of symptoms } \\
\hline Range & 3 months -8 years & 0.198 \\
\hline Mean & 3.6 years & \\
\hline \multicolumn{3}{|l|}{ Clinical stage } \\
\hline I & $22(46.8$ & 0.000 \\
\hline IIA & $18(38.3)$ & 0.0129 \\
\hline IIB & $7(14.9)$ & 0.0001 \\
\hline
\end{tabular}

There was a preponderance of females in the young age group. Twenty three out of 27 females were aged 30 while nine of the 20 males were aged 30 years and below. This difference was, however, not statistically significant $(p=0.02)$.

Twenty two $(46.8 \%)$ of the patients presented with clinical stage I disease, 18 cases $(38.3 \%)$ stage IIA and seven cases (14.9\%) stage IIB disease. None of the patients presented with stage III disease. There was no significant difference between sex and clinical stage of the disease $(p=0.03413)$. The young age group which accounted for 22 out of 35 patients aged 31 years and below were in clinical stage $I$. This difference was statistically significant $(\mathrm{p}<0.0000)$.

Five (20\%) out of 25 patients with clinical stage IIA or IIB presented with initial symptoms and signs of bulbar involvement. These included rhinolaliac (2), dyshpagia(1) and phonasthenia( 2 ). Four ( $16 \%$ ) of patients in stage IIA or IIB presented initially with a mixture of both ocular and bulbar features. The duration of symptoms from the onset of symptoms to the time of diagnosis varied from three months to six years with a mean of 3.6 years.

Table 3

Investigations

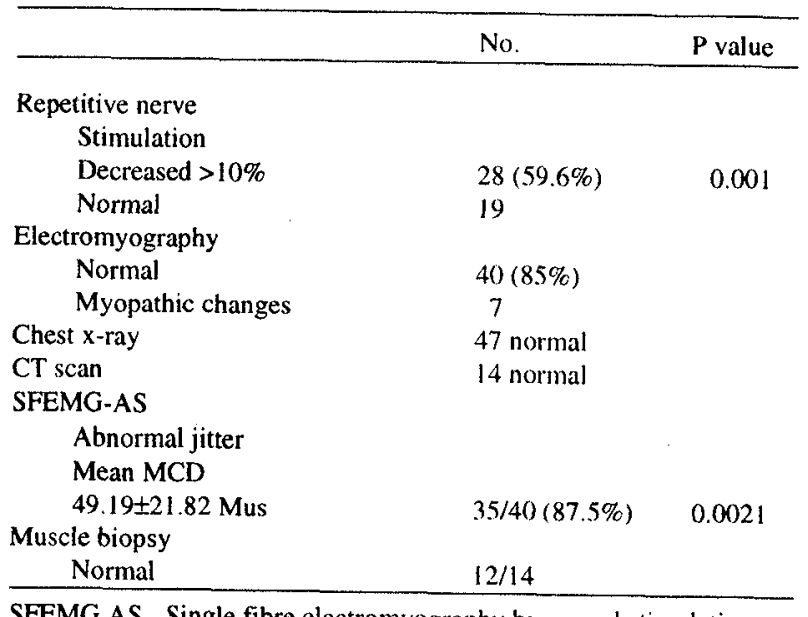

SFEMG AS Single fibre electromyography by axonal stimulation $M C D$ Mean consecutive differences
Table 3 shows the findings of the investigations. These were only performed on those patients who gave consent for these procedures. Repetitive nerve stimulation was positive in twenty eight $(59.6 \%)$ cases, which was significantly more in cases with stage IIA or IIB diseases than in stage $I(p=0.001)$.

Electromyography was normal in $49(85 \%)$ and only myopathic changes were seen in seven cases. Single fibre electromyography by axonal stimulation had abnormal jitter in $\mathbf{3 5}$ out $\mathbf{4 0}$ who consented for this test. Abnormal jitter was found to be significant in all patients with clinical stage II. In comparison $25(100 \%)$ patients in stage II had abnormal jitter compared with ten (45\%) patients in clinical stage I disease. This was statistically significant $(p<0.0001)$.

Fourteen patients gave consent for muscle biopsy out of 25 with clinical stage IIA and IIB disease. Of these 12 (86\%) had normal muscle, only two had mild muscle fibre atrophy. Three $(6.4 \%)$ of 47 patients had concomitant thyrotoxicosis.

\section{DISCUSSION}

Myasthenia gravis (MG) has been reported to be a rare disease in Africa(2-4). The results in our study lend support to this impression. The incidence of MG in our study is lower than those in Western Europe but almost similar to that reported in Libya(4). The low incidence in sub-Sahara Africa could be explained by probable lack of awareness on the part of the patients as well as health providers in recognising this disease. In our study the duration of symptoms varied from three months to six years with an average interval between onset of symptoms and diagnosis being 3.6 years; almost similar to that of patients reported in Libya(4). This is in contrast to Western Europe where patients present early, some within a few hours from the onset of symptoms(9). The incidence of MG was particularly low in both the under-five years and the elderly. Only two children were below ten years old and two patients were aged 60 years and above.

The mean age at presentation was 26.4 which was almost similar to European findings(1). The female-tomale ratio was 1.31:1 in our study, which was lower than that in China(10) and Libya(5), but similar to Europe where in one study the ratio was 1.4:1.9. The mean age for females with MG in our study was 21 years, which was much lower than the 26.5 years reported in Libya. The mean age for males was 34.2 years, thirteen years older than for females. This was similar to the European $(1,11,12)$ and other African(5) experiences.

In our study the mean ages for both females and males was lower than those reported in Europe(9) and in Libya(5). The peak mean age for late onset was slightly higher in males which supports the findings in Europe although only five of patients were above the age of 50 years in our study.

At the time of diagnosis $22(46.8 \%)$ patients had localised ocular MG, $17 \cdot(36.2 \%)$ patients had mild generalised disease and eight (17\%) patients had 
moderately severe generalised stage IIB. This stage of presentation was similar to that observed in some European studies $(1,8,11)$. In contrast, the Italian multicentre study by Mantegazza, and associates, over $90 \%$ of cases had generalised MG and only about five per cent had restricted ocular MG(12). Our study also differs from the Libyan study which had only one case out of eighteen cases with localised ocular MG(5).

Generalised MG with variable severity of stage IIA and IIB was found in 53.9\% (25 patients) in our study, similar to findings in the Hong Kong study among the Chinese(10). Bulbar involvement was the first indication in MG type II disease and presented as an ENT diagnostic problem, which has been reported elsewhere(13).

Three patients $(4.4 \%$ ) had other auto-immune diseases. This was lower than in other studies $(1,14)$. Anticholinesterase receptor antibodies test and other auto immune tests were not carried out in our study due to lack of facility. Nonetheless, it seems that the association of autoimmune disease and MG is rare in the African(3).

In our study, repetitive nerve stimulation (RNS) test was abnormal in $59.6 \%$ ( 28 out of 47 ), and SFEM was positive in $87.5 \%$ (35 out of 40 ). Chest $x$-rays were normal in all patients and CT scan was apparently normal in all 14 patients. It was apparent that SFEM was the most sensitive test of all the tests carried out. This is in agreement with Oh et al( 15$)$.

In conclusion, Myasthenia gravis is a rare condition in Tanzanian Africans. It is more common among young females than males. The mean age of presentation appeared 13 years later in male patients than in females. The average time interval between onset of symptoms and diagnosis was long. A generalised form of $\mathrm{MG}$ accounted for just about $50 \%$ of the patients as an initial presentation. A proportion of these $20 \%$ had initial bulbar clinical features. Ocular MG was common and accounted for about $47 \%$ of the patients. Repetitive nerve stimulation was abnormal in $59.6 \%$ and SFEM was abnormal in $87.5 \%$.

\section{ACKNOWLEDGEMENTS}

To the Director Genral, Muhimbili Medical Centre for permission to publish the findings of this study.

\section{REFERENCES}

1. Simpson J.A. Myasthenia gravis: In: Muscle Diseases (eds), J.N. Walton 1992; $628-665$.

2. Davidson J.C. Myasthenia Gravis in a Zambian African. Cent. Afr. J. Med. 1966:12:180-181

3. Tsega E., Choremi H., Buttazzo G.F. and Doncach D. Prevalence of autoimmune disease and autoantibodies in Ethiopia Trop. geogr: Med. 1980; 32: 231236.

4. Radhakrishnan K., Thacker A.I.C. and Maloo J.C. et cl. Descriptive epidemiology of some rare neurological diseases in Benghazi, Libya. Neuroepidemiology 1988 :159-164.

5. El-Zunni S., Prakash P.S., Saiti M. and Busnaina I.A. Myasthenia Gravis (MG): a preliminary repott. Cent. Afr. J. Med. 1996; 42:77-80

6. 1988 Population Census Volume 111 Dar es Salaam city.

7. Osserman K.E. Myasthenia gravis: Groune and Stratoon, New York, 1985.

8. Sorensen T.T. and Holm E.B. Myasthenia gravis in the Country of Viborg .Denmark. Eur. Neurol. 1989; 29:177-179

9. Newson-Davis J. Diseases of neuromuscular junction diseases of nervous system: Clinical neurobiology, WB Saunders Company: 2nd ed. 1992 Vol. 1:200

10. Yu Y.L., Hawkins B.R. Ip Ms, et al. Myasthenia Gravis in Hong Kong Chinese I. Epidemiology and adult disease. Acta. Neurol. Scand. 1992; 86:113-119.

11. Somnier F.E.. Keiding N. and Paulson O.B. Epidemiology of myasthenia gravis in Denmark. A longitudinal and comprehensive population survey. Arch. Neurol. 1991; 48:733-739.

12. Mantegazza R., Beghi E. and Pareyson D. et al. A Multicenter follow up study of 1152 patients with myasthenia gravis in Italy. $J$. Neurol. 1990; 237:339-394.

13. Salazar C., De Saa M. and Aparicio M., et al Myasthenia Gravis, Otorhinolaryngological considerations. Acta Otorrinolarinol. Esp. $2000 ; 51: 92-96$

14. Thorlacius S., Aortli J.A. and Riise T. et al. Associated disorders in myasthenia. Gravis; autoimmune diseases and their relation to thymectomy, Acta. Neurol. Scand. 1989; 80:290-295.

15. Oh S.J., Kim D.E. and Kuruoglu R. et al. Diagnostic sensitivity of the laboratory tests in myasthenia gravis. Muscle Nerve 1992; 15: $720-724$. 